\title{
RETRACTION
}

\section{Induction of tumor cell apoptosis via Fas/DR5}

\section{Wenzhu Li, Shengyu Wang, Caixia Chen and Guohong Zhuang}

Cellular \& Molecular Immunology (2013) 10, 184; doi:10.1038/cmi.2012.76.

Retraction to: Cellular \& Molecular Immunology 2006; Volume 3, Number 6, Page 467-471.

The corresponding author has indicated to the journal that this paper should be retracted as it is a duplicate publication. The author apologizes to the journal and its readers for any inconvenience caused. 